\title{
Utilizing Microscopy To Understand Mechanisms Of Heart Valve Morphogenesis
}

\author{
Lindsey J. Anstine ${ }^{1}$, Tori Horne ${ }^{1}$, Blair F. Austin ${ }^{1}$, and Joy Lincoln ${ }^{1,2}$ \\ 1. Center for Cardiovascular Research and The Heart Center at Nationwide Children's Hospital, \\ Columbus, $\mathrm{OH}$, USA \\ 2. Department of Pediatrics, The Ohio State University College of Medicine, Columbus, OH, USA
}

The prevalence of heart valve disease is estimated at $2.5 \%$ and this number is likely to increase with the steady rise in life expectancy and associated degenerative pathology. Affected individuals suffer significant morbidity and mortality and surgical intervention remains the only effective treatment. Disease of the valves can be congenital associated with structural malformations present at birth as a result of disturbances during embryonic valve development. Alternatively, phenotypes can be acquired later in life following sustained exposure to environmental risk factors including high cholesterol, tobacco-use, hypertension and aging, although there is increasing evidence to suggest that embryonic origins may also play a role.

Lineage fate mapping studies by our lab have shown that mature heart valve structures are largely derived from endothelial cells that undergo endothelial-to-mesenchymal transformation and give rise to endocardial cushion swellings in the atrioventricular canal and outflow tract regions. ${ }^{1}$ As embryonic development progresses, the newly transformed mesenchyme cells within the endocardial cushions migrate and proliferate at the distal tips to form elongated valve primordia structures. ${ }^{1}$ At this time, the endothelial-derived cells lose expression of mesenchyme markers and differentiate into embryonic valve interstitial cells (eVICs) where they mediate degradation of primitive extracellular matrix (ECM), and secrete specialized matrix components including collagen, proteoglycans and elastin. ${ }^{2}$

Formation of the mature valve leaflet is complete soon after birth and composed of organized ECM layers interspersed with VICs, and surrounded by a single layer of valve endothelial cells (VECs). It is the integration of extracellular and cellular components within the valve structures that maintain structure-function relationships throughout life. In contrast, diseased valves are characterized by alterations in matrix organization and composition, leading to biomechanical weakening and failure of the valve leaflets to open and close during the cardiac cycle. Many believe that these changes are mediated by the VIC population that express $\alpha$-smooth muscle actin in disease, suggesting a myofibroblast-like cell type. Furthermore, there is evidence to suggest that valve disease is associated with VEC dysfunction as evident by increased reactive oxygen species, and this in turn can modulate VIC phenotypes to promote pathological ECM remodeling. However, this is poorly understood.

To address this, we have initiated studies to define the role of VECs and VICs in maintaining healthy valve structures and further understanding how aberrations in these processes initiate disease. Using microscopy techniques, we showed that the VIC population is highly heterogeneous and phenotypes are dependent on age, species, location, and disease state. Furthermore, we identified phenotypic diversity across common models of mitral valve disease. ${ }^{3}$ These findings significantly contribute to characterizing the VIC population in health and disease and provide insights into the cellular dynamics that maintain valve structure in healthy adults and mediate pathologic ECM remodeling in disease states.

In addition to the VIC population, we examined the morphology and localization of VECs in health and 
disease and observed significant changes in mRNA profiles using RNA-seq analysis, and morphology by transmission electron microscopy. With age, a significant risk factor for valve disease, VECs exhibit decreased proliferation and cell-cell contact with neighboring endothelial cells on the surface of the valve (Figure 1), associated with increased permeability of the endothelium (Anstine et al., Unpublished). Therefore suggesting that increased infiltration of circulating risk factors (cholesterol, lipids, inflammatory cells) in the aging valve could promote pathogenesis.

We show that proliferation decreases with age, yet cell number is not significantly altered, and therefore the question is, how are valve cell populations maintained throughout life? Our preliminary studies show that extra-cardiac circulatory cells home into the valve endothelium and additionally contribute to the VIC population. We are currently investigating the engraftment of these bone marrow-derived cells into the valve cell populations and how this is influenced by age.

In summary, our studies have utilized microscopy to determine morphogenesis of heart valve structures during development, and characterize valve cell phenotypes after birth. Together, these studies provide a foundation for future studies determining how aberrations in embryogenesis and maturation contribute to the onset of valve disease.

\section{References:}

[1] Lincoln J, Alfieri CM and Yutzey KE. Developmental dynamics : an official publication of the American Association of Anatomists. 2004;230:239-50.

[2] Hinton RB et al, Circulation research. 2006;98:1431-8.

[3] Horne TE et al, Journal of cardiovascular development and disease. 2015;2:214-232.

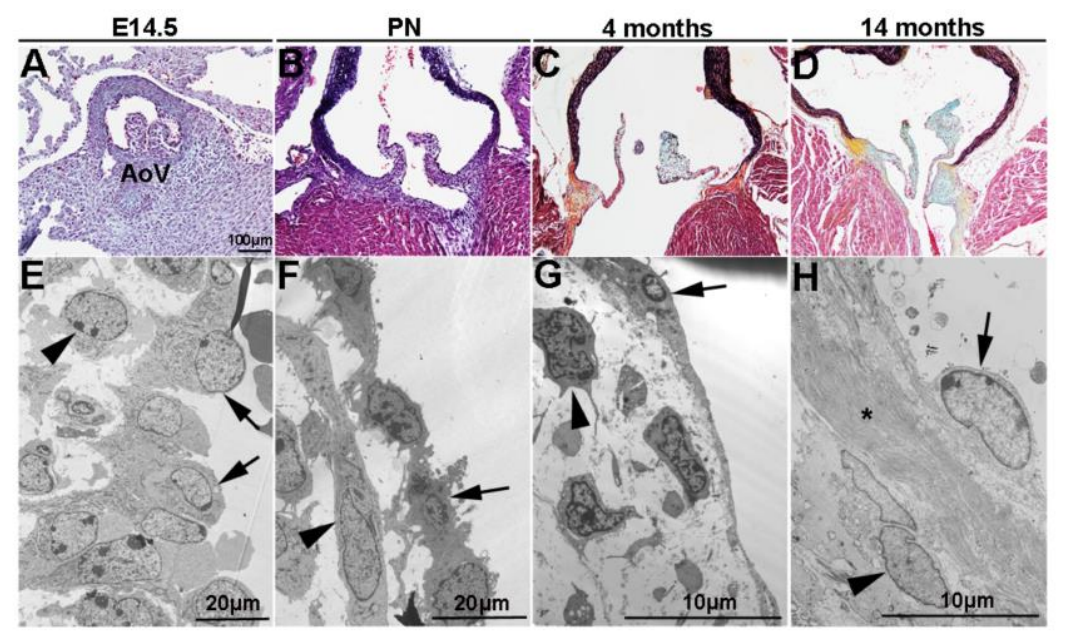

Figure 1. A-D, Pentachrome images of aortic valves at embryonic day $(\mathrm{E})$ $14.5(\mathrm{~A})$, post natal day $2(\mathrm{PN})(\mathrm{B}), 4$ (C) and 14 (D) months of age. E-H, Transmission electron microscopy to show valve endothelial cell (arrows) morphology and distribution in the valve at corresponding time points. Arrowheads indicate valve interstitial cells. 